SOME INSTANCES OF THE CONTRAST BETWEEN

\title{
DELIRIUM TREMENS
}

\author{
AND \\ INFLAMMATION OF THE BRAIN,
}

AS REGARDS THE
QUANTITY OF PHOSPHORIC ACID EXCRETED BY THE KIDNEYS.

BY H. BENCE JONES, M.A., F.R.S., FELLOW OF THE ROYAL COLLEGE OF PHYSICIANS, PHYSICIAN TO ST. GEORGE's hosPITAL.

Received December 17th, 1846-Read January 26th, 1847.

Рновphoric acid is excreted combined with the earths and with the alkalies. If lime or magnesia be present in sufficient quantity, then the whole of the phosphoric acid present in the urine is precipitated, on the addition of ammonia, in combination with these earths : but if an insufficient quantity of earthy matter be present, then the excess of phosphoric acid remains combined with potash or soda. Usually the lime and magnesia excreted by the kidneys are not equal to combine with above $\frac{1}{10}$ th part perhaps of the phosphoric acid in the urine. From $\frac{1}{4}$ th to $\frac{1}{18}$ th will be earthy phosphate, and the other $\frac{3}{4}$ ths to $\frac{17}{18}$ ths alkaline phosphate. Hence the variations in the quantity of earthy phosphate precipitated by ammonia will usually depend on the quantity of lime or magnesia passing through the system; and the sum of the alkaline and earthy phosphates must be determined if we desire to arrive at a knowledge of the quantity of phosphoric 
acid passing out of the system. In an extended inquiry* into the highest amount of phosphates excreted, it might have been anticipated that the determination of the lowest limit would be of equal interest with that of the highest; but very many analyses were made before the diminution of the phosphates in some cases of disease was made evident.

The following cases of delirium tremens present the lowest limit to the amount of phosphates; being in the lowest instance only 06 per 1000 urine, specific gravity 1017.9: whilst the cases of inflammation of the brain present the higher limit to the amount of phosphates excreted; being in the highest 13.38 per 1000 urine, specific gravity 1031.1: that is, 223 times less in the former case than in the latter, so far as a comparison may be made, the specific gravity and quantity of the urine not being taken into the account; and this comparison is the more admissible inasmuch as though the specific gravity of the urine is for the most part highest in inflammation of the brain, yet the quantity of urine in delirium tremens is usually excessively diminished.

It will be seen that in about twenty hours, in one case of inflammation, 58 ounces of water, specific gravity 1024.8, were excreted; whilst in a case of delirium tremens, in eleven hours the whole quantity excreted was 4 ounces, specific gravity $1019 \cdot 1$.

In the three cases which are here related of inflammation, the average quantity of phosphates is 8.26 per 1000 urine, specific gravity $1025 \cdot 3$.

During the acute stage of the three cases of delirium tremens, the average quantity of phosphates is only 67 per 1000 urine, specific gravity 1020.4.

Hence, then, sometimes at least, an analysis might determine whether delirium tremens or inflammation of the brain be present.

But it is not every case of delirium tremens which exhi-

- For the mode of analysis, see Philosophical Transactions, 1845 and 1846. 
bits this diminution; and it is not every case of inflammation of the brain which shows an increase of the phosphates. For if food can be taken in delirium tremens, the food furnishes phosphates, which hinders the diminution from being apparent; and if the inflammation of the brain be slight, the limits of variation in the healthy state rise so high that the slight increase, if present, cannot necessarily be considered as the result of inflammation. So that the chemical analysis can only be regarded as an assistance to the diagnosis, and not as alone sufficient to determine what the disease, in doubtful cases, may be. Such, at least, is the result so far as my experiments have hitherto gone.

The peculiar nature of the urine in delirium tremens is not less interesting to the physiologist than it is to the pathologist. Very frequently thick, scanty and high-coloured, it presents the characteristics of the most intense inflammation ; and when it is found that such urine may be acid for a week though containing almost no phosphoric acid, and. that it may contain so little phosphoric acid that this in one case was found to be insufficient to combine with above $\frac{1}{27}$ th of the earthy matter which was passing out of the system, it is probable that a more minute attention to such a state of secretion may lead to a more perfect understanding of the nature of the disease.

My intention, however, in the present paper is only to exemplify, 1st, that diminution, in the total amount of phosphatic salts excreted, which is to be observed in some cases of delirium tremens; and, 2ndly, to contrast therewith that increase which, in some cases of inflammation of the brain, is found to exist.

If any quick way of determining the amount of phosphates were possible, it would, without doubt, save much trouble, and add much to the usefulness of the distinction which I consider so diagnostic; but where quantities are in question we can judge only by weight or measure. Now, the measuring of the amount of a precipitate is always inaccurate ; and in the case of the amount of phosphates preci- 
pitated is not to be trusted even for rough approximations. Even the balance, from the difficulty of obtaining the substance to be weighed perfectly pure, gives only fine approximations. Hence all determinations of the quantity of phosphates present in the urine must be made with the balance; and the greater the care to obtain the substance to be weighed pure, the more valuable will be the results.

Case 1.-Ann Jones, ætat. 32, Queen's Ward, under Dr. Nairne, admitted on the 20th of May 1845, with delirium tremens, which had existed about four days, having had no sleep for two nights. She had a draught with a quarter of a grain of morphia every two hours. The following day she had had no sleep: she was not violent, but was constantly getting up in bed. She took forty drops of laudanum instead of the morphia. She continued the opium until she had taken five doses; half an hour after the last dose she went into a fit. She had been very violent previously; she bit her tongue, was convulsed, and then fell asleep for threequarters of an hour. She awoke very violent; but after some strong brandy-and-water she fell asleep and had a very quiet night, with much sleep.

The water passed in the afternoon was very small in quantity, acid; very thick on standing, from a brick-red sediment ; filtered, specific gravity $=1028 \cdot 7$.

\subsection{Grammes precipitated by Chloride of Calcium and Ammonia} gave 036 Phosphate of Lime.

Hence Phosphate of Lime $=1.07$ per 1000 urine.

22nd.-Manner now more quiet; much less tremor; face injected ; pulse 90, small; pupils small, but not excessively contracted. The opium was omitted, and she went out well on the 10th of June.

This case contains only a single analysis, but it shows a remarkable diminution of the phosphates; whilst the speeific gravity of the urine is as high as it usually is in inflam- 
mation of the brain. The quantity of water being in this case also very much diminished, a very small amount of phosphates was excreted during the time that the symptoms were most severe.

Case 2.-David Davis, an habitual drunkard, ætat. 35, hair-dresser, Hope Ward, under Dr. Nairne, admitted on the evening of the 18th of June 1845, having been in a sleepless state for three days. Reported to have had some anasarca for six weeks.

Two hours after admission he had a fit which lasted ten minutes; snorted and was convulsed; did not fall asleep for nearly an hour, during which time nothing could be made of him. At ten at night he had half a grain of morphia.

19th.-Very quiet during the night. Quiet this morning. Face injected. Answers sensibly; much tremor; urine was made at six in the morning, none having been passed before since seven in the evening; the whole quantity was four ounces, which, on cooling, gave a very pink sediment; when filtered, specific gravity $=1019 \cdot 1$.

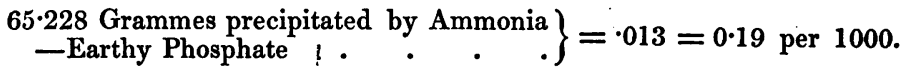
$\left.\begin{array}{l}\text { 32.999 Grammes precipitated by Chloride of } \\ \text { Calcium \& Ammonia-Phosphate of Lime }\end{array}\right\}=\cdot 081=2 \cdot 45$ per 1000.

Towards evening he began to be very restless; up and out of bed, and running about. Had no sleep and no food.

20th.-Extreme excitement all night. To-day very restless and excited. Urine passed from six in the evening till six the next morning, about seven ounces; specific gravity $=1019 \cdot 3$. A trace of albumen and few blood globules. Fibrinous cylinders uncertain.

$$
\begin{aligned}
& \left.\begin{array}{l}
\text { 49.949 Grammes precipitated by Ammonia } \\
\text {-Earthy Phosphate } \cdot . \\
\text { 33.007 Grammes precipitated by Chloride of } \\
\text { Calcium \& Ammonia-Phosphate of Lime }
\end{array}\right\}=\cdot 002=\cdot 04 \text { per } 1000 . \\
& \left.\begin{array}{l}
\text { 33.002 Grammes precipitated by Chloride of } \\
\text { Calcium \& Ammonia-Phosphate of Lime }
\end{array}\right\}=\cdot 004=\cdot 15 \text { per } 1000 .
\end{aligned}
$$


21st.-Confined all day; very violent; water drawn off at twelve; whole quantity, from six in the evening, about six ounces; pink sediment not so much as hitherto, strongly acid, and remained so for a week in an open vessel. Specific gravity $=1017 \cdot 9$.

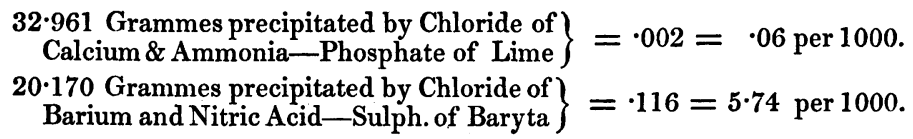

22nd.-In the evening he had two fits, and died.

Examination $15 \frac{1}{2}$-hours after death.-There were old and very extensive adhesions uniting the right lung to the sides of the chest. The lung itself, throughout the whole of its extent, was loaded with large quantities of red frothy serum, except at the back part of the lower lobe, where there was red hepatization and softening of texture. The left lung also was loaded, towards the back part, with red frothy serum, which ran out in large quantities when the lung was cut into. In both upper lobes there were, on the surfaces of the lungs, depressions with puckered margins, where the tissue of the lung was condensed and contained several tubercular deposits close to the depressions ; but no tubercular matter was found elsewhere. The heart was pale in colour and soft in texture ; all its cavities were dilated, but without hypertrophy of the walls. Both the aortic and mitral valves were slightly thickened and somewhat contracted; the other valves were healthy. The blood contained in the cavities of the heart was fluid and very thin. A few patches of atheromatous deposit existed at the root of the aorta.

The cavity of the peritoneum contained a small quantity of dark coloured serum. The liver was of a fawn colour throughout its whole extent. Its margins were round, and its surface presented a well-marked, granular appearance. When cut into, the structure of this organ presented a variegated colour from the condensation of the cellular tissue, which in several places had separated the acini and partially 
compressed them. But the condensation of the cellular tissue was not very great.

Both kidneys were large, much congested on their surfaces, and lobular; their capsules peeled off easily, and their surfaces presented, in several places, slight depressions, as if the cortical structure had there partially disappeared. In the other parts, the surfaces of these organs were perfectly smooth. The other organs in the abdominal cavity presented nothing remarkable.

The dura mater was more than usually adherent to the calvaria. The sub-arachnoid cellular tissue and the pia mater were filled with large quantities of clear fluid. The cineritious substance was pale, and the white substance presented slight venous congestion. The : ventricles were not enlarged. The structure of the brain appeared to be pretty healthy.

For this and all the post-mortem accounts I am indebted to Mr. Prescott Hewett.

This case is a more severe one than the preceding; the patient dying probably about the sixth day of the attack. The phosphates were found gradually to become less and less in quantity, until at last only 06 per 1000 urine, specific gravity $1017 \cdot 9$, were excreted. This is the smallest amount I observed in any of my experiments. The acidity of the urine, when scarcely any phosphoric acid was present, is worthy of remark. Here also is a good example of the diminution of the specific gravity, and of the quantity, of water made.

In eleven hours, 4oz. of water, specific gravity 1019.1

In twelve hours, 7oz. " " " 1019.3.

In eighteen hours, 6oz. $\quad " \quad$ " $\quad, 1017 \cdot 9$.

How far this is a conjoint effect of the delirium tremens, of disease of the liver, and of opium, I am not yet able to determine.

The following case shows a diminution of the specific gravity and of the amount of phosphates closely corresponding; and yet in it only 25 drops of laudanum had been taken when the analysis was made. 
This second case of delirium tremens is also very interesting, inasmuch as it closely agrees, as to the state of the lungs and the age of the patient, with the second case of inflammation of the brain which I am about to relate; yet how different is the amount of phosphates excreted. Two hundred and nineteen times as much in the latter case, if we compare the greatest quantity of the phosphates excreted in the one case with the least quantity in the other.

Case 3.-George Smart, ætat. 35, Fuller Ward, under Dr. Seymour. Admitted early in the morning of July 24th, 1845, having, at three, tried to strangle himself. At seven he slept about an hour, with 25 drops of laudanum. He has had six attacks of delirium tremens previous to this. The present began on the 19th: the patient having been drinking gin for three weeks, and having taken scarcely any food. Senna and salts were given on his admission. Water passed at one o'clock of deep reddish colour, about six ounces, acid. Traces of blood globules, albumen and fibrinous cylinders. Specific gravity $=1018 \cdot 0$.

65.292 Grammes precipitated by Ammonia $\}=\cdot 007=\cdot 10$ per 1000.

$\left.\begin{array}{l}\text { 32.965 Grammes precipitated by Chloride of } \\ \text { Calcium \& Ammonia-Phosphate of Lime }\end{array}\right\}=\cdot 003=\cdot 09$ per 1000 .

$\left.\begin{array}{l}\text { 32.965 Grammes precipitated by Chloride of } \\ \text { Barium and Nitric Acid-Sulph.of Baryta }\end{array}\right\}=\cdot 407=13.34$ per 1000.

To the clear ammoniacal fluid, from which all earthy phosphate had been precipitated, acid phosphate of soda was added. A very plentiful precipitate fell, which, under the microscope, was recognised to be chiefly phosphate of ammonia and magnesia. Thus proving the presence of magnesia :-

The 65.292 Grammes of the Ammonial fluid precipitated by Acid Phosphate of Soda, gave Earthy Phosphate $=\cdot 190$.

25th.-Has dozed a little. No sleep. Medicine was repeated every four hours. Has taken mutton and potatoes. Pulse 100, soft. Tongue furred, moist. Bowels acted twice last evening. Much perspiration. Very restless, continu- 
ally out of bed. Delirious. Urine said to have been plentiful during the night. This day the urine is acid, deep coloured. Specific gravity $=1022 \cdot 5$.

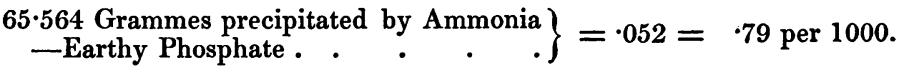
$\left.\begin{array}{l}\text { 33.111 Grammes precipitated by Chloride of } \\ \text { Calcium \& Ammonia-Phosphate of Lime }\end{array}\right\}=\cdot 202=6 \cdot 10$ per 1000 .

26th.-Pulse very weak. Tongue furred. Pupils very small. Perspiration excessive. But little sleep. Forty drops of laudanum every six hours.

27th.-Slept last evening and all night. Water drawn off at twelve ; deep colour, acid. Gave a very considerable precipitate of urate of ammonia; filtered. Specific gravity $=1027 \cdot 9$.

33.284 Grammes precipitated by Chloride of Calcium and AmmoniaPhosphate of Lime $=\cdot 280=8 \cdot 41$ per 1000 urine.

The following day, specific gravity $=1022 \cdot 9$.

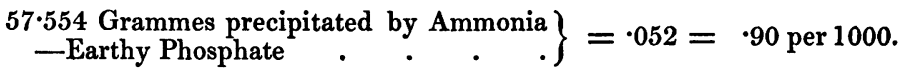
$\left.\begin{array}{l}\text { 33.122 Grammes precipitated by Chloride of } \\ \text { Calcium \& Ammonia-Phosphate of Lime }\end{array}\right\}=\cdot 231=6.97$ per 1000 .

He remained in the house till the 20th of August, quite free from all delirium, tremor, or restlessness, suffering only from an injury.

In this case, examination of the phosphates was made when only 25 drops of laudanum had been taken, yet the amount was diminished to $\cdot 10$ per 1000 urine, specific gravity 1018.0. So little phosphoric acid and so much magnesia were present, that $\frac{1}{27}$ th part only of the magnesia in the urine was precipitated on the addition of the ammonia. From the quantity of sulphuric acid present, and from the patient having taken salts and senna, the greater portion of this excess of magnesia doubtless came from the medicine.

The following day food was taken, and then the amount of phosphates increased, and the urine began to lose the characteristics of delirium tremens. 
This case, taken with the previous one, shows the course which the variations in the phosphates follows. As the disease increases, no food being taken, the phosphates diminish more and more. As the case improves, and food is taken, the phosphates rise until the healthy amount is excreted.

In the second case of inflammation of the brain scarcely any food was taken, certainly not more than in the second case of delirium tremens; and yet the phosphates will be seen passing off in larger quantities than in the healthy state, when food and exercise can be taken.

Case 4.-James Mileham, ætat. 28, waiter, admitted under Dr. Nairne, Jan. 29, 1845, into King's Ward, having been ill about a fortnight, and having had pectoral symptoms for a year.

30th.-Tongue was thickly furred, dry and brownish. Face very red. Skin hot and dry. Pulse 70, full, soft. Pupils rather large, the right a little the larger. Bowels confined. Answers slowly, sometimes rightly, sometimes wrongly; says he sees but one thing at a time now, but did see double. Seldom answers questions the first time of asking. Head very hot. Delirium during the night. Water was passed at 7 с. м. before any food was taken; deep-coloured, clear, acid; gave a precipitate with heat, which re-dissolved with acid. Specific gravity $1025 \cdot 3$.

$$
\begin{aligned}
& \text { 66.389 Grammes precipitated by Ammonia } \\
& \left.\begin{array}{l}
\text { Earthy Phosphate } . \cdot . \\
\text { 33.202 Grammes precipitated by Chloride of } \\
\text { Calcium \& Ammonia-Phosphate of Lime }
\end{array}\right\}=\cdot 060=\cdot 90 \text { per } 1000 . \\
& \left.\begin{array}{l}
\text { 33.202 Grammes precipitated by Chloride of } \\
\text { Calcium \& Ammonia-Phosphate of Lime }
\end{array}\right\}=\cdot 227=6.83 \text { per } 1000 \text {. }
\end{aligned}
$$

31st.-Skin and head hot. Pulse 90, full and strong. Tongue very coated, brown, dryish. Face red. Pupils as yesterday. Takes very little food. Looks less heavy. Urine passed at 11 A..M., clear, strongly acid, gave a precipitate of urate of ammonia on standing. Specific gravity $=1028 \cdot 1$. 
$\left.\begin{array}{c}\text { 66.606 Grammes precipitated by Ammonia } \\ \text {-Earthy Phosphate . . . . . }\end{array}\right\}=\cdot 099=1 \cdot 48$ per 1000 .

$\left.\begin{array}{l}\text { 33.2.91 Grammes precipitated by Chloride of } \\ \text { Calcium \& Ammonia-Phosphate of Lime }\end{array}\right\}=\cdot 330=9 \cdot 91$ per 1000 .

$\left.\begin{array}{l}\text { 33.290 Grammes precipitated by Chloride of } \\ \text { Calcium \& Ammonia-Phosphate of Lime }\end{array}\right\}=\cdot 326=9 \cdot 79$ per 1000 .

Feb. 2nd.-Pulse 92, much smaller. Tongue brown, dry. Face still flushed. Pupils more dilated. No strabismus. Feeling about for things, and wandering. No sleep at all. Talking, singing and spitting. Much more heavy, and seemingly incapable of answering. Urine passed at $2 \frac{1}{2}$ P. M., strongly acid. Specific gravity $=1024 \cdot 8$. Continued acid for a month.

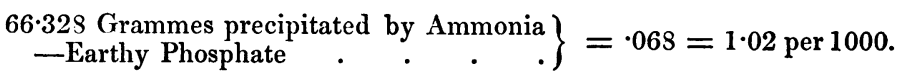

$\left.\begin{array}{l}\text { 33.184 Grammes precipitated by Chloride of } \\ \text { Calcium \& Ammonia-Phosphate of Lime }\end{array}\right\}=\cdot 280=8 \cdot 43$ per 1000 .

3rd.-Face not so flushed. Right eyelid more drooping than left. Right pupil more dilated than left. Some strabismus. Pulse small, rapid, 120. Has been roaring all night. Skin hot and dry. Tongue dry and brown. Water was passed last evening. At $2 \frac{1}{4}$ P.M. to-day, fifty-eight ounces of urine were drawn off, clear, strongly acid. Specific gravity $=1021 \cdot 2$. Continued acid for three weeks.

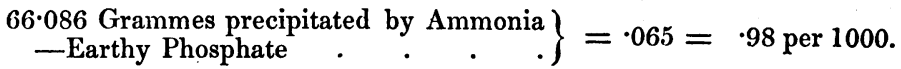

$\left.\begin{array}{l}\text { 33.068 Grammes precipitated by Chloride of } \\ \text { Cal }\end{array}\right\}=\cdot 191=5 \cdot 77$ per 1000 .

4th.-Tongue very coated, protruded to the left side. Pulse 120, small. Skin hotter than yesterday. Face flushed, mouth dry. Strabismus more than yesterday. Right pupil the most dilated. More quiet, less wandering. Urine drawn off at $10 \frac{1}{2}$ A. M., about 24 ounces, acid to test paper, clear. Specific gravity $=1016 \cdot 9$.

\footnotetext{
$\left.\begin{array}{c}\text { 6.5.670 Grammes precipitated by Ammonia } \\ \text {-Earthy Phosphate . . . . . }\end{array}\right\}=\cdot 041=\cdot 62$ per 1000.

$\left.\begin{array}{l}32 \cdot 930 \text { Grammes precipitated by Chloride of } \\ \text { Calcium \& Ammonia-Phosphate of Lime }\end{array}\right\}=\cdot 140=4 \cdot 25$ per 1000 .
} 
Urine drawn off in the evening, about 14 ounces, gave a plentiful deposit on standing. Specific gravity $=1024 \cdot 6$, acid.

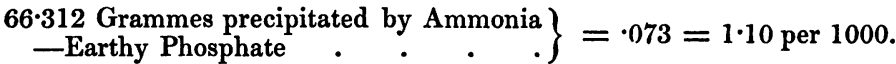

33.177 Grammes precipitated by Chloride of $\}=.235=7 \cdot 08$ per 1000

Calcium \& Ammonia-Phosphate of Lime $\}=\cdot 235=7 \cdot 08$ per 1000.

5th.-Quite quiet, no wandering. Knew his brother this morning. Pulse 160, not so small as yesterday. Tongue very brown and dry, but put out when the patient was told. Right pupil dilated to the uttermost. He took some tea at 3 P. M., which he asked for, became a great deal more heavy, and died at 7 P.M.

Examination eighteen hours after death.-Membranes of the brain perfectly healthy, on the upper and lateral surfaces; but at the base, especially about the floor of the third ventricle and the fissure of Sylvius, the sub-arachnoid cellular tissue and the pia mater were very much thickened by an effusion of yellow, concrete, semi-transparent lymph. Convolutions of the brain flattened, and sulci somewhat effaced. Both the grey and the white substances of the brain were of a decidedly pink colour. This discoloration of the grey substance existed principally in that part which is next to the white substance. Whilst the discoloration in the latter substance was disposed in bands and streaks, giving the white substance a marbled appearance. Ventricles of the brain distended by a very large quantity of transparent fluid. Septum broken down and flocculent in about its middle; but the neighbouring parts of the fornix and the corpus callosum were certainly not softer than natural. Arteries at the base of the brain healthy.

There were old and firm adhesions partially uniting the right lung to the walls of the chest. On the left side the adhesions were universal and very firm. Both lungs were, throughout their whole structure, thickly studded with miliary tubercles and semi-transparent grey granules; the intervening structure being much congested and somewhat softer than natu- 
ral. In different parts of the lungs were several vomicæ of various sizes, the largest being in the apex of the right lung. Pericardium, heart, and coagula, healthy.

Liver and spleen healthy. Two or three small tubercles were found in the cortical structure of the kidneys, which were otherwise perfectly healthy. Several ulcerations destroying the mucous membrane were found in the caput coli; but the other parts of the intestines presented nothing worth noticing.

This case, dying about the 22nd day, is by no means a marked example of the most acute inflammation of the brain. The amount of the phosphates and the specific gravity of the urine are rather high; but the most striking difference from delirium tremens is in the amount of water secreted. In about eighteen hours there were $\mathbf{5 8}$ ounces of water, containing as much phosphates as $5 \cdot 77$ per 1000 urine, specific gravity 1021.2. In the succeeding thirty hours at least 38 ounces of urine were drawn off.

The occurrence of inflammation of the brain, in phthisical patients, quite unconnected with tubercles in the brain, although stated and illustrated by Dr. Abercrombie, has not frequently occurred to $M$. Louis. In two years $I$ have seen in St. George's Hospital seven instances of such a complication. They were all patients about the age of this man; all were examined after death; and the existence of tubercles in the lungs, and no tubercles in the brain, was proved.

Case 5.-John Hitch, brickmaker, æetat. 36, very intemperate, admitted July 17, 1844, into Hope Ward, under Dr. Nairne. Had been for two months an out-patient for cough and spitting. Has complained of his head, and been lightheaded for eight days. The face is flushed, the tongue furred, and the pulse slow. Want of all respiration, with dullness at the back of the right lung, and moist rales, and want of healthy respiration at right, apex. He was with difficulty induced to take medicine.

20th.-Pulse 60, large and compressible. Seems heavy, VoL: $X X X$. 
but does as he is told. Lies on his back. Takes no food, only porter.

The following day he was quite insensible; not answering and picking the bed-clothes. Some strabismus. Urine drawn off, deep coloured, acid, deposited much urate of ammonia : filtered, specific gravity $=1029 \cdot 7$.

66.684 Grammes precipitated by Ammonia $\}=\cdot 122=1.82$ per 1000 .

$\left.\begin{array}{l}\text { 66.684 Grammes precipitated by Chloride of } \\ \text { Calcium \& Ammonia-Phosphate of Lime }\end{array}\right\}=\cdot 877=13 \cdot 15$ per 1000 .

22nd.-Still strabismus. Left pupil dilated, right contracted. Takes no notice; lies on his back, breathing hard. Pulse at times 65. Passes his evacuations in bed. Muttering delirium.

23rd.-Pulse varying from 70 to 85 . Strong beating of the carotids. Ptosis of the left eye-lid. The right eye seems to be moved easily, the left eye remains fixed. Moves both his legs and arms. Excessive perspiration. Water drawn off at 10 A. M., deep coloured, acid, no sediment. Specific gravity $=1033 \cdot 0$.

66.900 Grammes precipitated by Ammonia $\}=\cdot 155=2 \cdot 31$ per 1000 .

$\left.\begin{array}{l}\text { 33.443 Grammes precipitated by Chloride of } \\ \text { Calcium \& Ammonia-Phosphate of Lime }\end{array}\right\}=\cdot 405=12 \cdot 11$ per 1000 .

25th.-Tongue dry. Pulse 120, large, soft and regular. Face very flushed. Left eye quite closed, and pupil immoveable ; right eye injected. Lies muttering. Face more wasted. Urine deep coloured, acid. Specific gravity $=1030^{\circ} 0$.

$\left.\begin{array}{c}\text { 66.708 Grammes precipitated by Ammonia } \\ \text {-Earthy Phosphate. } . . .\end{array}\right\}=\cdot 090=1 \cdot 35$ per 1000.

$\left.\begin{array}{l}\text { 33.354 Grammes precipitated by Chloride of } \\ \text { Calcium \& Ammonia-Phosphate of Lime }\end{array}\right\}=\cdot 318=9 \cdot 53$ per 1000 .

26th.-Face less red. Breathing harder. Right pupil more dilated. Pulse 118, not so strong, regular. Much muttering. Tongue dry, brown. Sordes on the teeth. Back beginning to slough.

27th.-In the evening he died. 
Examination thirty-eight hours after death.-On the upper part of the head there were some adhesions of the opposed surfaces of the arachnoid, rendering the separation of the dura mater difficult. The structure of the brain was peculiarly firm and tenacious; especially the upper portions of both hemispheres. The ventricles of the brain were much distended with transparent serum. The cerebral substance forming the walls of both lateral ventricles was much softened for the depth of a couple of lines. The fornix and septum lucidum especially were so soft that their natural structure could no longer be recognized. Towards the lower part of the anterior lobe, upon both sides, the medullary substance of the brain also was broken down, and some portions had assumed the consistence of thick cream. The arachnoid membrane at the base and over the pons was rendered opaque by the effusion of serum into the cells of the pia mater. The pia mater generally was congested.

The apices of both lungs were condensed, and presented several small, oval, chalky deposits, mixed with numerous small masses of tubercular deposit. On the right side were old adhesions of the pleura costalis and pulmonalis, between which recent effusions of serum and lymph had taken place.

This is another more acute case exemplifying the connection between phthisis and inflammation of the brain. The quantity of phosphates and the specific gravity of the urine were remarkably increased: 13.15 per 1000 urine, specific gravity 1029.7 ; and 12.11 per 1000 urine, specific gravity 1033.0. This forms a most marked contrast with the second case of delirium tremens. The smallest possible quantity of food was taken by this patient, so that the amount of phosphates must be considered as uninfluenced thereby.

Case 6.-John Esterling, ætat. 36, stableman, Fuller Ward, under Dr. Seymour, admitted June 28, 1846. Said to have had a cough nine months; to have been ill six weeks, and bad in his head nearly three; complaining of pain and giddiness. 
On admission, very stupid and stubborn; knew no one; complained of pain in his head, especially the back part. Face flushed. Sleeping constantly; could scarcely be awoke to take anything; and when he did so was constantly sick. Sees distinctly. Pulse jerking, 44. Has eaten nothing for two days.

C. C. nuchæ ad そ̌xii. Cal. gr. iij. Antim. gr. iij. Pil. Saponis C. gr. ij. 6tis horis. After cupping, the pulse fell to 40.

30th.-Mouth was very sore, and since it has become so he has not slept so much, and has recognised his wife. Pulse 54. More easily roused. Still constant sickness. Small quantity of urine passed about 7 P.M. : specific gravity $=1031 \cdot 1$, acid, giving a thick pinkish deposit.

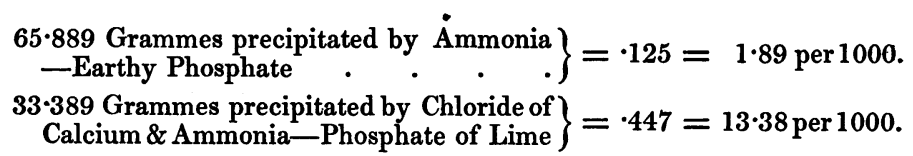

July 1st.-Omit Calomel. No sickness until the evening; then only once.

2nd.-Face flushed. Pulse 70, compressible, jerking. Doubtful whether he sees correctly. Little difficulty of hearing. Tongue furred. Mouth very sore. Skin cool. Head hot. Sleeps quite quietly, without wandering; but talks and laughs to himself. Passed his motions in bed during the night. More rational to-day than he has been since his admission. Ten ounces of water passed at 7, slight deposit, acid : filtered, specific gravity $=1022 \cdot 9$.

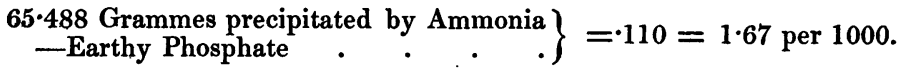

$\left.\begin{array}{l}\text { 33.123 Grammes precipitated by Chloride of } \\ \text { Calcium \& Ammonia-Phosphate of Lime }\end{array}\right\}=\cdot 200=6.03$ per 1000 .

3rd.-Much more rational.

5th.-Emp. Lyttæ inter scapulas.

8th.-Pulse 90. Skin cool. Still a little pain in the head. 
9th.-Pulse soft, 62. Has been up. Urine passed early in the morning, acid, clear, yellow colour. Specific gravity $=1016.3$.

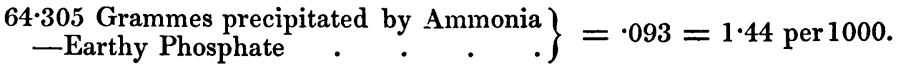

$\left.\begin{array}{l}32 \cdot 909 \text { Grammes precipitated by Chloride of } \\ \text { Calcium \& Ammonia-Phosphate of Lime }\end{array}\right\}=\cdot 092=2 \cdot 79$ per 1000 .

From this time he rapidly improved, and went out in his ordinary state of health on the 16th of August.

In this case there were also pectoral symptoms, but the existence of tubercles could not be established by the stethoscope. Soon after his admission, when the symptoms were very urgent, the amount of phosphates and the specific gravity of the urine were much increased; as the symptoms yielded to treatment the phosphates decreased. Taken in connection with the two.preceding cases, this points out the course which the variations of the phosphates observe in inflammation of the brain.

\section{Conclusion.}

Such then is the contrast which delirium tremens and inflammation of the brain sometimes exhibit as regards the amount of phosphates excreted.

A most marked diminution occurs in the one set of cases and a considerable increase in the other. A similar striking contrast is seen in the treatment of these diseases; the one requiring the most active antiphlogistic remedies, the other being aggravated by them. Judging from the treatment, we conclude phrenitis to be inflammatory, and we consider delirium tremens as not in the smallest degree partaking of the nature of inflammation. Hence the excretion of the excess of phosphates may be regarded as resulting from inflammatory action going on in the brain, whilst the diminution of the same phosphates in delirium tremens must be considered as caused by the positive hindrance of that process of formation of phosphoric acid which in the healthy state is continually taking place. 
Perhaps the following might be given, as at present the nearest approximation that can be made to the connection of these facts. That in the healthy state, a portion of the phosphoric acid which is excreted results from the action of the inspired oxygen on the phosphorus of the phosphorized fatty matter of the brain. In delirium tremens, either from the action of the substances which cause this state of disease, or from the peculiar state of the nervous structure, this production of phosphoric acid by the action of oxygen is almost entirely hindered; whilst in inflammation of the brain, it seems probable from these experiments that an increased formation of phosphoric acid results from the inflammation of the nervous structure. In other words, it seems necessary to admit that inflammatory action and the action of oxygen must be in some close relation to each other;-a relation which is also brought before us in the increase of temperature of inflamed parts, and in the peculiar oxides of albumen which form the chief constituents of the inflammatory crust of the blood. The admitted influence of the action of oxygen on life would lead us to expect that it would be an important element in disease.

The excess of action, or the want of action, of the socalled vital gas may, possibly, ultimately be proved by the balance, not only to be traceable by its effects on the nervous tissue, but other tissues may give a corresponding result. With this view the variations of the sulphates in disease deserve the most careful investigation. 